\title{
Commentary
}

\section{On the Nature, Assessment, and Treatment of Fetal Pain: Neurobiological Bases, Pragmatic Issues, and Ethical Concerns}

Anita Gupta, DO', and James Giordano, $\mathrm{PhD}^{2}$

From: ${ }^{1}$ Johns Hopkins University Medical Center, Baltimore, MD; ${ }^{2}$ Georgetown University Medical Center, Washington, DC, and Samueli Institute, Alexandria, VA.

${ }^{1}$ Dr. Gupta is a Fellow, Department of Anesthesiology, Program in Pain Medicine, Johns Hopkins University Medical Center, Baltimore, MD.

${ }^{2}$ Dr. Giordano is Scholar in Residence, Center for Clinical Bioethics, Associate Professor, Division of Palliative Medicine Georgetown University Medical Center Washington, DC 20057 and Director, Program in Brain, Mind, and Healing Research, Samueli Institute, Alexandria, VA,

Address correspondence: Dr. James Giordano Center for Clinical Bioethics and Division of Palliative Medicine

Georgetown University Medical Center 4000 Reservoir Rd, Bldg D, Rm 238 Washington, DC, 20057

Email: jgiordano@neurobioethics.org

Disclaimer: This work was supported, in part, by funds from the Laurence Rockefeller Foundation, and Samueli Institute (JG).

Conflict of interest: None

Free full manuscript: www.painphysicianjournal.com
Over the past 2 decades, the issue of fetal pain and stress has attracted considerable attention and has become the focus of ongoing debate in light of advances in medical diagnostics and technology that allow invasive prenatal medical intervention(s), and an enhanced understanding of fetal neurophysiology, together with a broadened appreciation for the relationship of brain and consciousness. In this essay, we explore the issue of fetal pain based upon knowledge of both the ontogeny and function of the nervous system and the interaction of brain, mind, and pain. We posit that contemporary understanding of neurological development fortifies appreciation that pain sensation and perception occurs during (later) fetal life and that this pain warrants clinical consideration during the conduct of invasive prenatal procedures. We present differing perspectives on this issue, discuss the relative merits and difficulties of these positions, and ultimately describe the fundamental basis for a consideration of fetal pain and address this consideration with regard to pragmatic and ethical benefits, burdens, and risks. We adopt a neurocentric, yet consilient approach that entails both scientific and philosophical orientations. By attempting to reveal inherent limitations in our contemporary appreciation and approach(es) to fetal pain, we hope to illustrate the need for and posit potential venues toward resolving these limitations and dilemmas.

Key words: ontogeny, fetal pain, intervention, analgesia, neurobiology, ethics

Pain Physician 2007; 10:525-532

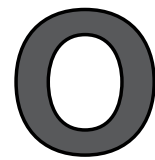

ver the past 2 decades, the issue of fetal pain and stress has attracted considerable attention and has become the focus of ongoing debate. An enhanced understanding of fetal neurophysiology, together with a broadened appreciation for the relationship of brain and consciousness, has instigated pragmatic and ethical concerns about the capacity for the fetus to experience pain. The question of fetal pain is not simple however, as any approach to this issue must account for pain as a neural process and as sensation, the perceptibility of noxiousness in the fetus and the proximate and more durable effects of pain - as neural event and experience - on the subsequent development and activity of the nervous system and the potential for lifelong cognitive-emotional-behavioral effects in 
the person in whom that nervous system exists and functions.

By acknowledging that the fetus is indeed able to sense and experience pain (i.e. is "painient") (1), and that the effects of pain are deleterious (both in the short and long term) we cannot avoid challenges posed by the questions of

1) how such pain could and should be assessed or considered,

2) what constitutes the "best" approaches and techniques to treat fetal pain, and

3) the relative benefits, burdens and/or risks to both fetus and mother that such treatment(s) could incur.

Obviously, epistemological consideration of fetal pain also gives rise to speculation about the importance of neural processes to consciousness, the relation of conscious processes to painience and sentience, neurally-based explanations of the moral status of the fetus, and the need to regard painience as a basis for defining the moral responsibility to others (1-3). In light of this, it becomes apparent that the question of fetal pain, while obviously important in pain medicine, equally situates pain medicine to assume a leading philosophical, ethical and pragmatic role in influencing and guiding social attitudes that reflect our most current understanding of brain, mind, self and suffering.

In this essay, we explore the issue of fetal pain based upon a progressive knowledge of both the ontogeny and function of the nervous system, and the interaction of brain, mind and pain. We posit that an enhanced understanding of neurological development, coupled with a growing body of empirical evidence, fortifies appreciation that pain sensation and perception occurs during (later) fetal life, and that this pain warrants clinical consideration during the conduct of invasive prenatal procedures. We present differing perspectives on this issue, discuss the relative merits and difficulties of these positions, and ultimately describe the fundamental basis for a consideration of fetal pain and address this consideration with regard to pragmatic and ethical benefits, burdens and risks (both immediately and more distally). Our focus is un-apologetically neurocentric, but this analysis is none the less consilient, entailing both scientific (e.g. genetic, embryological, pharmacological, toxicological, bio-psychosocial) and philosophical (i.e. epistemic, anthropologic, and ethical) orientations to the issues, problems and solutions. By attempting to reveal inherent limitations in our contemporary appreciation and approach(es) to fetal pain, we hope to illustrate the need for, and posit potential venues toward resolving these limitations and dilemmas.

\section{Defining the Issue}

Recent advances in prenatal imaging, analytic, and surgical procedures now allow in utero diagnosis and treatment of a number of medical conditions (4). Yet, despite the hundred year history of anesthesia as a staple measure in almost every surgical intervention (5), the provision of anesthesia and/or analgesia to the fetus during such procedures remained unconsidered, based upon the longstanding belief that the fetus was insensate to pain (6), reflecting an inadequate understanding of the structure and function of the nervous system during prenatal development. However, by the mid- to late-1980s, this bias became increasingly contested. During those years, a large body of literature elucidated the functional maturation of nociceptive and pain-modulatory systems during gestation and in neonates using a variety of animal models that were predictive of pre- and early post-natal neural structure and function in humans (7-15). These studies, as well as a number of behavioral, neurophysiologic and anatomical investigations of pain responses in human prenates and infants were summarized by Anand and Hickey (16), whose seminal work on pain in the human fetus and neonate

1) acknowledged that the neural pathways for pain are intact and viable during late gestation, and

2) established the first clear statement for the need to address and treat neonatal, if not fetal pain, as well.

It should be noted, however that Anand and Hickey's paper appeared in 1987, and while its relevance to the treatment of neonatal pain was obvious and important (17), at that time there were few procedures that would be likely to produce fetal pain. Of course, the work raised significant practical and ethical debate regarding the provision of analgesics or anesthetics during late gestational termination of pregnancy (moral issues beyond that of fetal pain notwithstanding - a discussion of which is beyond the scope of this paper). However, the increasing sophistication of diagnostic technologies and surgical capabilities over the 20 years has made prenatal medical intervention more viable and common (18), and this technical and technological progress re-engages the need to consider evidence that more precisely defines the ability of the fetus to sense and experience pain, 
and thus provides meaningful direction in the medical obligations to treat such pain.

\section{Developmental Meurobiology of Nociceptive and ANTI-NOCICEPTIVE SYSTEMS}

\section{The Nociceptive System}

Much of the information regarding the ontogeny of the nociceptive and anti-nociceptive axes is gained from studies using the rat model, and is based upon the observation that the first postnatal week in the rat is developmentally equivalent to the last gestational trimester in the human (19). The neural systems that subserve pain develop progressively and differentially throughout ontogeny (7-15). Peripheral free nerve endings and primary nociceptive afferents develop early, with A-delta fibers becoming functional prior to C-fibers $(10,11,13)$. However, central connectivities within the spinal cord, and the ascending spinothalamic tract, while anatomically present early in development, do not appear to be functional until somewhat later (i.e. the first postnatal week in the rat, viz. - the last gestational trimester in the human) $(20,21)$. Both peripheral and spinal components of the nociceptive pathways exhibit a rostro-caudal maturation (22), such that the forelimbs (i.e. fingers and hands) and face develop prior to the posterior torso and/or legs.

What is less clear is when and how the supraspinal pain system(s) develop. Unlike the spinal pathways, supratentorial development tends to follow a somewhat more caudo-rostral pattern (22), and forebrain structures generally mature later than hind- and/or midbrain loci. The reticular-midbrain, hypothalamicpituitary, thalamo-cortical, and many limbic pathways are in place and active by the last gestational trimester $(23,24)$. Moreover, the developmental pattern of early transcriptional factors, most notably c-fos, suggests that there is inherent phenotypic plasticity of the system that may respond to in utero conditions, pathological states, and/or pharmacological manipulation $(25,26)$.

But yet unresolved questions remain as to exactly what systems subtend the sensation and noxious experience of pain, whether each and/or all of these systems, networks, and pathways need to be fully functional for the pain experience to occur, and if not, is the fetal experience of "pain" equally or equivalently noxious as that of the infant, child or adult?

\section{Conflicting Evidence?}

It has been suggested that the commonly accepted measures of fetal glucocorticoid levels and EEG activity represent non-specific responses to stress, are only circumstantial justification for any consideration of fetal pain, and are therefore inconclusive (27). The core of this argument is that the fetal stress response is insufficient evidence of pain sensation or perception. Given the definition of "pain" as "...an unpleasant sensory and emotional experience..."(28), then for a subjective phenomenon of noxious sensation to be "experienced" as "pain" it would necessarily involve the substrates, and have some combinatory qualities of sensation, cognition, and emotion (29). To be sure, in the child, adolescent and adult "pain" is a multi-dimensional event that entails lower, intermediate and higher conscious processes $(30,31)$, and the "meaning" of pain involves and is derived from "... unquestionably a sensation in a part of the body.... and an emotional experience..."(28). Chugani and Phelps have posed that if specific areas of the cortex that are responsible for the associative aspects of linking sensation to cognition and emotion are not fully developed until after birth (32), then the fetus cannot "experience" pain qua pain.

We object to these arguments on several grounds. First is that these positions fail to acknowledge the most current perspective(s) regarding the structurefunction relationship of nervous systems; these can be colloquially summarized using a computer analogy to posit that the presence of the "wetware" (i.e. the neural substrates) is often a reliable predictor that the "program" (i.e. pain) can and will be executed. This is strengthened by robust comparative studies of mammalian species as anatomical, physiological and behavioral models of human morphology, development, neurophysiology and pain $(7-15,33)$. But even so, what is more important is that we have come to recognize that the "experience" of pain, while involving a number of higher neuroanatomical areas (e.g. the somatosensory and associative cortices), appears to be reliant upon subcortical structures. Furthermore, these structures likely do not function site specifically, but are engaged as and within a network, the properties of which contribute to mental process(es), and which may vary as a consequence of geno- phenotype interactions, time and development in each individual $(30,31)$. Thus the second flaw in the arguments that attempt to negate the possibility of fetal pain is that they tend to attribute the function of the whole brain 
to particular parts (i.e. the mereological fallacy). Third, these arguments make the erroneous assumptions that pain is uniform across individuals, and is "knowable" only in terms of its meaning and the linguistic capacities used to describe it. Clearly this is incorrect; both the sensation and experience of "pain" are individually variant, subjective, and knowable only to the one in pain. We cannot "know" what the feeling(s) of pain are like for another person; rather we can only comprehend the explanation of their first person experience $(30,31,34)$. Yet, the mere lack of linguistic capability does not refute the possibility that other individuals can have pain. If this were the case, we would not acknowledge that pain occurs in those who cannot speak, the profoundly mentally impaired, the demented (or animals), because of the fact that they do not "describe" their pain, per se. In essence, we cannot "know" what it is like to be another being (35); rather we can only recognize or "know" others' experiences by relating them to our own subjective experience(s) $(36,37)$. We do this by

1) objectifying others' experiences by applying intellectual knowledge of some common processes that are equivalent or similar in ourselves and others (i.e. phenomenological relativism),

2) perception of some set of knowable reactions, responses, and/or semiotics, and/or

3) the direct explanation of subjective experience(s) by others.

Given that the existential reality of "being a fetus" cannot be explained, a frame of reference does not exist for the prenatal condition, therefore we must rely on our understanding of the nervous system, as well as our interpretations of neurophysiologic data and behavioral responses to develop a contemporary hermeneutics that allow appreciation of fetal experience. Neurophysiologic findings taken together with the observed nociceptive responses of premature human infants (from 25 through 42 gestational weeks) seemingly confirm that pain occurs in the pre- and neonate, that there is considerable plasticity in the system, and these observations support consideration of providing analgesia to the fetus undergoing some noxious invasive medical procedure (38-40). Or do they?

\section{Anti-nociceptive Systems}

Pain and pain-modulation in the intact organism are complex processes of spatial and temporal interactions within and between anatomical and neuro- chemical systems that function as multi-directional, hierarchical, networks. While poly-threshold tactile capabilities develop relatively early, in so far as to be functionally mature at birth (to facilitate maternal contact, object acquisition, orientation and learning), it is not clear whether pain modulatory systems are equivalently developed prior to, or at birth. Generally, the capacity for modulating low- to moderate threshold stimuli develops during the last trimester, and like the tactile system, seems to first involve the forelimbs and face. The ability to mitigate higher threshold noxious input occurs later, and may not fully mature until well after birth (22,41-44). A number of factors may subserve this pattern. First is the maturation of anatomical substrates of the analgesic neuraxis. Neuronal-, neural-glial-, and tract- integrity all progress at different rates and achieve functional maturity at different prenatal (and sometimes postnatal) time points. Similarly, metabolic systems responsible for the biosynthesis of neurotransmitters and neuromodulatory agents, as well as the pre- and post-synaptic receptors that mediate anti-nociceptive effects do not develop uniformly. Perhaps most importantly, it must be borne to mind that phenotypic expression occurs as a consequence of environmental exposure and experience, and the prenatal in utero environment is both relatively stable and non-noxious (at least under non-pathological conditions). So while the anatomical structures for pain modulation may be in place during the last trimester and at birth, it could be that these systems are not necessarily active, but rather that they are "primed" for the subsequent stimulation that will determine their expression, and this somewhat "delayed" functional development of components of the pain modulatory system(s) may reflect an absence of noxious input in utero $(22,45,46)$. But what if pathology and/or invasive procedures to correct such pathology (prematurely or abnormally) activate these systems?

\section{Pragmatic Issues, Ethical Concernes}

As Barr notes, the "...critical question, therefore, is not when analgesia develops..." but rather is "... how the physiological systems become organized to mediate analgesia at different ages (19)."Perhaps this speaks to an even larger question, namely how neural (and other physiological) systems become organized, on the whole. Edelman's conceptualization of "...neurons that fire together, wire together" (47), taken together with accumulating evidence for the functional and structural plasticity of peripheral and central ner- 
vous systems, as well as "top-down" effects that such plasticity incurs (viz. - in endocrine, immune, cardiovascular systems, etc.), underscores the sensitivity of these substrates - both as individual components, and as functional networks - to changes induced by the internal and/or external conditions (29-31).

In light of this, we pose five questions as critical to any discussion of fetal pain and whether it should be treated: First, does the diagnosed pathological condition affect the life potential or potentiality of the fetus significantly enough to warrant invasive medical intervention? Second, does the pathologic disturbance negatively affect the developmental trajectory and function of the pain and pain modulatory systems so as to produce significant (predisposition or occurrence of) pain? Third, if we advance this line of thought, then we are compelled to question whether we may need to reduce pain incurred by the pathology and/or its treatment. This prompts the fourth question of how to assess fetal pain, and the fifth of whether such pain can be treated. While answers to the first and perhaps second question "evolve" from an objective focus upon the fetal pathology and its effects, any account that acknowledges fetal pain must address the inherent problem associated with both assessing such pain, and using this (and other) information as necessary to develop concrete protocols and paradigms for fetal pain therapeutics.

As previously discussed, at very best we must employ neurophysiologic data from the studies of animals and premature human neonates, together with a working philosophical construct of the brain-mind relationship to the experience of pain to form a rational grounding for evaluating fetal pain. Simply put, we must presume that what is painful in the neonate or developing animal will be painful to the human fetus, and ascribe some scale of noxiousness to quantify these experiences.

But even given this practical approach to the problem of assessing pain in the prenate, we must still confront the issues that surround effectively treating fetal pain. Pharmacokinetic variables (e.g.- maturational differences in hepatic and neural enzymes, blood-brain/blood -CSF barriers, etc.) affect the ability and action profile of a variety of drugs that could be used to induce analgesia and anesthesia in the fetus, and while these maturational distinctions are generally understood (19), considerable individual variation exists, and this variation may be further affected by pathological state(s). As well, pharmacological ma- nipulation can affect enzymatic and even structural factors to alter the subsequent organizational development and induce short term and long term functional changes. Simply, we must ask how could and will the decision(s) to treat or not to treat affect the resultant trajectory of the developing fetus? If we do not treat prenatal pain, are we predisposing the system to progressive sensitization that could be expressed as a lifelong diathesis for neuropathic pain? On the other hand, can we be sufficiently precise in the dose calculations and delivery of prenatal analgesics or anesthetics so as not to incur pharmacologic de-sensitization and a predisposition for tolerance, loss of therapeutic effect, need for continuous dose-escalation and possibilities of increased adverse side effects? Moreover, how can we predict what effect(s) prenatal (or even neonatal or childhood) administration of neurotropic agents will have upon the developing nervous system, and the cognitive, behavioral and/or emotional manifestations that may occur as a result?

\section{Of Mother and Fetus...}

And what of the benefits, burdens, and risks to the mother? Yet even this line of inquiry remains enfranchised to the well being of the fetus, for if we speculate upon fetal pain in the context of distress, then we must also contemplate how maternal stress can affect the fetus. Is there any hard and fast "rule" that provides guidance to when we could or should treat the fetus and not the mother, or vice versa? While the doctrine of double effect appears to be a likely candidate for such guidance (48), how are we to accurately assess the potential goods, burdens or harms that might be incurred to the fetus by treating or not treating in light of our current understanding (or lack thereof) of the putative long-term effects that pathology or pharmacological manipulation might have on the developing nervous system? Given our contemporary knowledge of neural development (and its implications for the nature of brain, mind and self), it would seem that we are pragmatically and morally compelled to at least consider providing the fetus with some regard for the clinical care of pain and suffering.

Ultimately, the gravid female, prima facie, presents as 2 patients - mother and fetus (at very least during the last trimester) - whose practical and moral worth are the subjects of clinical care. Therefore, we argue that the right and good treatment that affords well being to both mother and fetus is of primary concern, and cannot be arbitrarily differentiated. We acknowl- 
edge, however, that situations occur that force circumstantial discriminations in the type and level of care that is provided to mother and fetus. How can such clinical decisions be made? Clearly, the needs for pain care differ for the adult mother and the fetus, and these differences must be accounted for by the facts and impressions that underlie any reflection upon the circumstances, intentions, actions and consequences that contribute to clinical decision-making.

\section{Toward Resolution: Reflection, Rules, and the Roles of Phronesis and Research.}

Obviously, no single dimension of knowledge or decisional skill will provide universal answers or abilities. Maintaining thorough, up-to-date knowledge of the development, structure and function of the neural systems that mediate pain and analgesia is vital, but we opine that it is equally important to recognize how and why this objective information contributes to, and "fits" into a contextual understanding of

1) how these systems create first-person experience(s), and this being the case,

2) how pain medicine (like other forms of medicine, as well) must deal with the uncertainty of applying objective fact(s) to the realities of others' subjective experience.

In actuality, medicine remains a science of probabilities (49), and its humanistic domains are executed as a hermeneutic exercise (50). As such, just as any single dimension of knowledge cannot completely serve these functions (51), we have argued elsewhere and re-claim here that the use of a single ethical approach is equally constraining (52-55).

We have posited that there are certain "rules" that define the practice of pain medicine and these are built upon and shaped by the facts of pain, realities of pain medicine, and its practical and moral claims $(55,56)$. But such "rules" cannot, and do not provide the answers for each and every dilemma in all situations. At this particular point in time, the specifics of whether and how to provide fetal pain care must be addressed and decided upon using a case by case method. However, to re-iterate our previous work, irrespective of the rules that shape the practice and/or the utility of the casuistic approach, the final decisional process relies upon, and underscores the therapeutic and moral agency of the individual physician as steward of knowledge to uphold the medical fiduciary (52-56). We have argued the importance of prudence (i.e. phronesis, practical wisdom), as the cardinal, fulcral virtue that allows the use of diverse types of knowledge to inform and direct morally sound action(s) (52-54).

At least in part, the knowledge that contributes to such prudence (i.e. - the factual basis of what pain is, and the capabilities of medicine, in general, and pain medicine more specifically) is dependent upon, and reflects advances in research $(57,58)$. Research findings contribute to not only the scientific aspects of pain medicine, but must compel and sustain advances in medical education, guidelines, and the ethical and policy frameworks that shape clinical practice. To be sure, there are no "easy resolutions" to the questions we have raised relevant to the issues in fetal pain care. As our scientific knowledge and technology advance, the capabilities of what can be done make ardent strides, and it becomes increasingly apparent that the true goal of research is to enable prudence so as to provide insight to how such advances should be utilized to fulfill the humanitarian purpose of medicine. Our ongoing work is committed to these studies.

\section{Acknowledgments}

This work was supported, in part, by funds from the Laurence Rockefeller Foundation, and Samueli Institute (JG). The authors acknowledge the assistance of Sherry Loveless in the preparation of this manuscript.

\section{References}

1. Ryder R. Painism: A Modern Morality. Centaur, London. 2001.

2. Anand KJ, Carr DB. The neuroanatomy, neurophysiology, and neurochemistry of pain, stress, and analgesia in newborns and children. Pediatric Clinics of 5 North America. 1989; 36.4: 795-822.

3. 109th Congress, U.S.A. "Unborn Child
Pain Awareness Act of 2005." 1st Session, S.51. (January, 2005).

4. Farmer DL. Fetal surgery: a brief review. Pediatric Radiology. 1998; 28.6: 409413.

5. Magner LN. A History of Medicine. Taylor and Francis, Boca Raton. 2005.

6. Derbyshire, SWG. Fetal "pain"- a look at the evidence. American Pain Society Bulletin. 2003; 13(4).

7. Bronstein, DM, Mittledorf, P, Sadeghi MM, Mitteldorf P, Sadeghi MM, Kirby $\mathrm{K}$, Lytle LD. Visceral nociception in developing rats. Dev Psychobiol 1986; 19:473-487.

8. Fitzgerald M. The post-natal develop- 
ment of cutaneous afferent fibre input and receptive field organization in the rat dorsal horn. J Physiology 1985; 364: 1-18.

9. Fitzgerald M. The development of activity evoked by fine diameter cutaneous fibres in the spinal cord of the newborn rat. Neuroscience Letters 1988; 86(2): 161-166.

10. Fitzgerald M. A physiological study of the prenatal development of cutaneous sensory inputs to dorsal horn cells in the rat. J Physiology 1991; 432: 473482.

11. Fitzgerald M, Gibson S. The postnatal physiological and neurochemical development of peripheral sensory $\mathrm{C}$ fibres. Neuroscience 1984; 13: 933-944.

12. Fitzgerald M, King AE, Thompson SWN, Woolf CJ. The postnatal development of the ventral root reflex in the rat; a comparative in vivo and in vitro study. $\mathrm{Neu}$ roscience Letters 1987; 78: 41-45.

13. Leong SK, Sheih JY, Wong WC. Localizing spinal cord projecting neurons in neonatal and immature albino rats. $J$ Comp Neurology 1984; 228:18-23.

14. Semba E, Shiosaka S, Hara Y, Inagaki S, Sakanka M, Takatsuki K, Kawai Y, Tohyama $\mathrm{M}$. Ontogeny of the peptidergic system in the rat spinal cord: Immunohistochemical analysis. The Journal of Comparative Neurology 1982; 208 : 5466.

15. Pickel VM, KK Sumal, Miller RJ. Early prenatal development of substance $P$ and enkephalin-containing neurons in the rat. J Comp Neurology. 1982; 210 (4): 411-422.

16. Anand KJ, Hickey PR. Pain and its effects in the human neonate and fetus. New England Journal of Medicine. 1987; 317(21): 1321-1329.

17. Schechter NL. The undertreatment of pain in children: An overview. Pediatric Clinics of North America. 1989; 36 (4): 781-794.

18. Coakley FV. Role of magnetic resonance imaging in fetal surgery. Topics in Magnetic Resonance Imaging. 2001; 12(1):39-51.

19. Barr GA. The ontogeny of nociception and antinociception. In: Thadani P. (ed.) Biological Mechanisms and Perinatal Exposure to Abused Drugs. NIDA Research Monograph 158, NIDA, Baltimore, MD. 1995, p 172-201.

20. Miya DY. The Development of the Lateral Spinothalamic Tract and Its Re sponse to Lesion. Ph.D. Dissertation, City University of New York, NY 1993.

21. Miya DY, Barr GA Prenatal development of the lateral spinothalamic tract in rats. Neurosci Abstr 17:1158, 1991.

22. Giordano J, Barr GA. Morphine- and ketocyclazocine-induced analgesia in the developing rat: Differences due to type of noxious stimulus and body topography. Dev Brain Res 1987; 32:246-253.

23. Mrzljak L, Uylings, HBM, Kostovic I, van Eden CG. Prenatal development of neurons in the human prefrontal cortex: I. A qualitative Golgi study. I Comp Neurology 1988; 271: 355-386.

24. Meaney MJ, Tannenbaum F, Francis D, Plotsky PM. Early environmental programming hypothalamic-pituitary-adrenal responses to stress. Seminars in Neuroscience 1994; 6 (4): 247-259.

25. Williams S, Evan G, Hunt SP. Spinal cfos induction by sensory stimulation in neonatal rats. Neuroscience Letters 1990; 109: 309-314.

26. Yi DK, Barr GA. The Induction of fos-like immunoreactivity by noxious thermal, mechanical and chemical stimuli in the lumbar spinal cord of infant rats. Pain. 1995; 60 (3): 257-265.

27. Giannakoulopoulos X, Sepulveda W, Kourtis P, Glover V, Fisk, NM. Fetal plasma cortisol and beta-endorphin response to intrauterine needling. Lancet. 1994; 344: 77-81.

28. International Association for the Study of Pain. IASP Pain Terminology. 2004. Available at: https://www.iasp-pain. org/terms-p. html. Accessed June 20, 2007.

29. Grahek, N. Feeling Pain and Being in Pain. MIT Press, Cambridge, MA, 2007.

30. Giordano J. Understanding pain as disease and illness- Part One. Prac Pain Management. 2006, 6(6): 70-73

31. Giordano J. Pain as disease and illnessPart Two. Prac Pain Management. 2006; 6(7): 65-68.

32. Chugani HT, Phelps ME. Maturational changes in cerebral function in infants determined by $18-f-D G$ positron emission tomography. Science 1986; 231: 840-843.

33. Chapman CR., Loeser JD. (eds.) Issues in Pain Measurement: Advances in Pain Research and Therapy, Volume 12. Raven Press, NY, 1989.

34. Jaspers K. Vernunft und Existenz. Johs. Strom Verlag, Bremen 1949.
35. Nagel T. Mortal Questions. Cambridge University Press. Cambridge, 1979.

36. Grondin J. Gadamer's basic understanding of understanding. In: Dostal RJ. (ed.) Cambridge Companion to Gadamer. Cambridge University Press, 2002, p. 36-51.

37. Dilthey W. Gesammelte Schriften: V. Band: Die Geistige Welt, Einleitung in Die Philosophie Des Lebens. B.G. Teubner Verlag, Stuttgart, 1990, p 303-317.

38. Williams C. Framing the fetus in medical work: Rituals and practices. Social Sci Med 2005; 60: 2085-2095.

39. Rosen MA. Anesthesia for fetal procedures and surgery. Yonsei Med Journal 2001; 42: 669-680.

40. Craig KD, Witfield M.F., Grunau RV, Linton J, Hadjistavropoulos HD. Pain in the preterm neonate: Behavioural and physiological indices. Pain 1993; 52: 287-299.

41. Hughes HE, Barr GA. Analgesic effects of intrathecally applied noradrenergic compounds in the developing rat: Differences due to thermal vs mechanical nociception. Brain Research 1988; 469 (12): 109-120.

42. Kehoe P, Blass EM. Conditioned opioid release in ten-day-old rats. Behavioral Neuroscience 1989; 103: 423-428.

43. Leslie F.M., Loughlin S.E. Development of multiple opioid receoptors. In: Miller MW. (ed.) Development of the Central Nervous System, Effects of Alchohol and Opiates. Wiley-Liss, NY, 1992.

44. Zhang AZ, Pasternak GW. Ontogeny of opioid pharmacology and receptors: high and low affinity site differences. Eur J Pharmacology 1981;73 (1): 29-40.

45. Giordano J, Barr GA. Effects of neonatal spinal cord serotonin depletion on opiate-induced analgesia in tests of thermal and mechanical pain. Brain Research 1988; 469: 121-127.

46. Giordano, J. Anti-nociceptive effects on intrathecally administered 2-methylserotonin in developing rats. Devel. Brain Res 1997; 98: 142-144.

47. Edelman GM. Wider Than the Sky: The Phenomenal Gift of Consciousness. Yale University Press, New Haven, 2004 .

48. Sulmasy, DP, Pellegrino ED. The rule of double effect: Clearing up the double talk. Arch Internal Medicine. 1999; 159 (6): 545

49. Dogra N, Giordano J, France N. Cultural 
diversity teaching and issues of uncertainty: the findings of a qualitative study. BMC Medical Education 2007; 7( 8).

50. Svenaeus F. Hermeneutics of medicine in the wake of gadamer: the issue of phronesis. Theoretical Medicine and Bioethics. 2003; 24: 407-431.

51. Giordano J. On knowing: Domains of knowledge and intellectual virtue in practical pain management. Prac. Pain Management 2006; 6: 65-67.

52. Giordano J. Agents, intentions and actions: moral virtue in pain medicine. Prac. Pain Management 2006; 6(4): 7680.
53. Giordano, J. Moral agency in pain medicine: Philosophy, practice and virtue. Pain Physician, 2006; 9:71-76.

54. Giordano J. Pain, the patient and the physician: Philosophy and virtue ethics in pain medicine. In: Schatman M. (ed.) Ethics of Chronic Pain Management. NY, Taylor-Francis, NY. 2006.

55. Maricich Y, Giordano J. Pain, suffering and the ethics of pain medicine: Is a deontic foundation sufficient? Am. J. Pain Management, 2007; 17: 130-138.

56. Giordano J. Technology, techniques and tekne: On the ethical use of guidelines in the practice of interventional pain medicine. Pain Physician, 2007; 10: 15.

57. Giordano J. Pain research: Can paradigmatic revision bridge the needs of medicine, scientific philosophy and ethics? Pain Physician 2004; 7: 459463.

58. Giordano J. Good as gold? The randomized controlled trial: pragmatic and ethical issues in pain research. Am. J. Pain Management 2006; 16; 68-71. 